\title{
VISUALIZAÇÃO DE ONTOLOGIAS: estudos e perspectivas
}

\author{
Cristiane Mendes Netto* \\ Gercina Ângela Lima*
}

\begin{abstract}
Apresenta os resultados de uma revisão sistematizada de literatura que teve como objetivo analisar os estudos e as perspectivas de trabalhos futuros sobre visualização de ontologias. A metodologia foi desenvolvida com aplicação de etapas do processo Proknow-C (Knowledge Development Process - Constructivist) para seleção de um portfólio bibliográfico de 35 referências científicas, publicadas de 2007 a 2017. No resultado da análise, identificou-se quatro abordagens principais de estudo do tema: apresentação de ferramentas e protótipos; comparação de ferramentas; estudos com usuários e revisão de literatura, sendo que $57 \%$ dos trabalhos selecionados eram destinados à apresentação de ferramentas/protótipos. Quanto a perspectivas de trabalhos futuros em visualização de ontologias, o estudo aponta quatro temáticas: visualização de ontologias complexas, onde há centenas de elementos; ferramentas para usuários leigos em ontologias; estudos de usabilidade e de requisitos de usuários e avaliação de contribuição cognitiva para usuários. A contribuição deste trabalho se alicerça no delineamento deixado às futuras pesquisas e na legitimação do processo de revisão sistemática como um gerador de conhecimento.
\end{abstract}

Palavras-chave: Visualização de Informação. Ontologias. Revisão Sistemática.

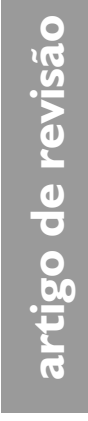

* Mestre em Ciências da Computação pela Universidade Federal de Minas Gerais, Brasil. Professora da Universidade Vale do Rio Doce, Brasil. Doutoranda no Programa de Pós-Graduação em Gestão e Organização do Conhecimento da Universidade Federal de Minas Gerais, Brasil.

E-mail: cris.netto@gmail.com.

** Doutora em Ciências da Informação pela Universidade Federal de Minas Gerais, Brasil. Pós-doutorado no Departamento de Biblioteconomia y Documentación da Universidad Carlos III, Madrid, Espanha. Professora associada da Escola de Ciência da Informação da. Universidade Federal de Minas Gerais, Brasil. Docente permanente no Programa de Pós-Graduação em Gestão e Organização do Conhecimento da Universidade Federal de Minas Gerais, Brasil.

E-mail: limagercina@gmail.com.

\section{INTRODUÇÃO}

$\mathrm{N}$ o campo da organização da informação e do conhecimento, os estudos de ontologias têm tratado principalmente das suas aplicações como instrumento de representação do conhecimento de domínios. Pode-se considerar que o interesse comum desses estudos reside no potencial que as ontologias possuem de fornecerem uma conceituação compartilhada de um domínio para ser comunicada a pessoas e a sistemas computacionais.

No âmbito computacional, a criação de ontologias tem sido beneficiada pelo desenvolvimento de linguagens formais, como a Web Ontology Language - OWL, e de ferramentas sofisticadas como o Protégé, que oferecem os recursos formais para profissionais especialistas em ontologias. No entanto, dada a complexidade dos do- mínios a serem representados, as ontologias usualmente podem conter centenas e até milhares de classes e instâncias. Essa estrutura pode se tornar de difícil visualização e extremamente complexa para a compreensão humana.

Nesse contexto, têm-se buscado contribuições da área de Visualização de Informação. Essa área é definida por Card; Mackinlay e Shneiderman (1999) como o uso de representações de dados abstratos suportadas por computador e interativas para ampliar a cognição. Para Freitas et al (2001), a Visualização de Informação é uma área de aplicação de técnicas da computação gráfica, geralmente interativas, visando auxiliar o processo de análise e compreensão humana de um conjunto de dados, por manipulações das representações gráficas.

Com o propósito de auxiliar o uso de ontologias e a compreensão humana do 
domínio representado por elas, a visualização de ontologias tem sido abordada na literatura científica das áreas de Computação e de Ciência da Informação. Com o objetivo de identificar os tipos de estudos e perspectivas de trabalhos futuros nessa temática, apresentam-se neste artigo os resultados de uma revisão sistemática de literatura. Sua contribuição se alicerça no delineamento deixado às futuras pesquisas e na legitimação do processo de revisão sistemática como um gerador de conhecimento.

A organização do artigo apresenta-se da seguinte forma: a Seção 2 descreve o referencial teórico. A metodologia da pesquisa é apresentada na Seção 3. Em seguida, são abordadas, na Seção 4, os resultados e, na Seção 5, as considerações finais.

\section{REFERENCIAL TEÓRICO}

As ontologias são definidas por Gruber (1993) como especificações explícitas de uma conceituação. Ao abordar essa definição, Vickery (1997) descreve que a conceituação significa uma visão abstrata do domínio que se deseja organizar para algum propósito. Portanto, a ontologia pode ser comparada a um esquema conceitual para um sistema, que fornecerá uma descrição lógica para compartilhamento dos dados. Na organização do conhecimento, uma ontologia fornece a representação de um domínio, possibilitando a criação de uma base de conhecimento que incluirá o conhecimento simbolicamente representado para resolver problemas específicos ou responder consultas sobre o domínio.

Nesse sentido, Campos (2010) considera que as ontologias estabelecem um vocabulário comum para uma comunidade que precisa compartilhar informação em um determinado domínio de um modo que as definições podem ser interpretáveis computacionalmente. Isso inclui representações de conceitos e relações. Sobre a conceituação proporcionada pelas ontologias, apresenta que essa pode ser entendida como uma abstração, uma visão simplificada do mundo, que se representa com o propósito de auxiliar na compreensão, compartilhamento ou consenso sobre uma área de conhecimento. As definições formais possibilitam que agentes de software possam estabelecer inferências para dar suporte à manipulação do conhecimento explicitado em uma ontologia. Com o objetivo de auxiliar os usuários no uso de ontologias, pesquisas na área de visualização de informação passaram a ser aplicadas a esse campo.

O processo de visualização de informação está relacionado com a transformação de dados abstratos em gráficos ou imagens, denominados visualizações. Assim, o objetivo na construção das visualizações é explorar a capacidade de percepção humana para que, a partir das relações espaciais exibidas, o usuário interprete e compreenda as informações apresentadas e, ainda, deduza novos conhecimentos.

Em um estudo de técnicas de Visualização de Informação para construção de interfaces para Sistemas de Recuperação de Informação - SRI, Correa e Vieira (2013) concluem que as informações representadas por meio de recursos visuais são de grande importância na comunicação dos SRI e podem auxiliar o usuário a entender sua estrutura e organização ao possibilitar a interação com as informações e a descoberta de novos conhecimentos.

Nesse contexto, pode-se considerar que essa área visa à representação de dados abstratos por meio de recursos computacionais em uma forma de exibição que amplie a cognição humana. Na evolução histórica da área, observa-se que o primeiro registro do termo Visualização de Informação é encontrado em Card; Robertson e Mackinlay (1991). Em seguida, apresentam-se os estudos de Feiner e Beshers (1990) e Shneiderman (1996). O desenvolvimento da área tem seguido mutuamente influenciado por estudos de múltiplas áreas, tendo como objetivo comum transformar dados abstratos em representações visuais que possam auxiliar o entendimento e a descoberta de informações.

Segundo Shneiderman (1996), a apresentação de dados de forma gráfica maximiza o aproveitamento da capacidade de percepção da visão humana, além de permitir que os usuários encontrem mais facilmente padrões, agrupamentos, lacunas ou tendências em dados ou, ainda, que descubram características escondidas em um grande volume de dados. Segundo o autor, a Visualização de Informação permite a exploração mais conveniente da informação por meio de uma linguagem visual. Pesquisadores e designers de interface do usuário devem buscar métodos de visualização de informação que ofereçam uma integração mais intuitiva da tecnologia com a tarefa de recuperação de informação. 
A visualização de informação aplicada à ontologia consiste em representar graficamente os elementos que constituem as ontologias: classes, relações, axiomas e instâncias, com possibilidades de realização de tarefas de interação sobre esses elementos. Com base nas características predominantes, oferecidas pelas ferramentas de visualização de ontologias, Katifori et al. (2007) agruparam os métodos de visualização de ontologias nas seguintes categorias:

a) Lista identada: uma lista identada apresenta a taxonomia da ontologia em uma estrutura semelhante à organização de pastas do Windows Explorer.

b) Árvore de links e nós: na árvore de links e nós a ontologia é representada como um conjunto de nós interconectados (grafos) que geralmente podem ser expandidos e retraídos pelo usuário.

c) Visualização Zoomable: uma visualização baseada em aproximação (zoomable) apresenta os nós filhos aninhados dentro de seu nó pai e permite ao usuário fazer ampliação para e sair dos nós.

d) Preenchimento de espaço: a vista de preenchimento de espaço apresenta os nós subdividindo o espaço da tela. O tamanho de cada subdivisão depende dos atributos do nó, como o número de nós secundários.

e) Foco e Contexto: essa visão apresenta contexto e foco ao mesmo tempo exibindo o nó em foco ampliado e o resto dos nós colocados em torno dele.

f) Paisagem 3D: a visão da paisagem da informação apresenta objetos 3D codificados por cor e tamanho em um plano usando a metáfora da paisagem.

Mediante o exposto, perante a diversidade de modelos para visualização de ontologias e da complexidade dos aspectos do processo de transformar dados em visualizações, destaca-se a relevância deste estudo de revisão de literatura, com propósito de oferecer uma visão geral do cenário de pesquisa nessa temática, com vista ao desenvolvimento da área de organização da informação e do conhecimento.

\section{METODOLOGIA}

No que se refere ao objetivo deste trabalho, esta pesquisa se caracteriza por ser exploratória e descritiva. Segundo Gil (2010), a primeira característica se apresenta em pesquisas que têm como objetivo proporcionar maior familiaridade com um problema, com vista a torná-lo mais explícito, envolvendo o aprimoramento de ideias ou a descoberta de intuições. A pesquisa descritiva visa a descrição das características de determinada população ou fenômenos, ou o estabelecimento de relações entre variáveis.

Quanto ao delineamento da pesquisa, no que se refere ao planejamento em sua dimensão mais ampla, ela classifica-se como pesquisa bibliográfica, desenvolvida com base em material já elaborado, constituída de trabalhos científicos (GIL, 2010). A abordagem da análise dos dados baseia-se na pesquisa indutiva, em que, partindo-se de dados particulares constatados, infere-se uma verdade geral, não contida nas partes examinadas (MARCONI; LAKATOS, 2010).

A natureza da pesquisa é aplicada, tipo que, segundo Marconi e Lakatos (2003), caracteriza-se por seu interesse prático, isto é, que os resultados sejam aplicados, imediatamente, na solução de problemas que ocorrem na realidade.

Como instrumento para sistematização da revisão de literatura, utilizou-se o processo Proknow-C (Knowledge Development Process Constructivist) proposto por Tasca et al (2010). A identificação desse processo ocorreu a partir de pesquisas em periódicos nacionais da área de Ciência da Informação, entre os quais estão os trabalhos de Ensslin; Ensslin e Pacheco (2012), Ensslin et al (2015) e Machado et al (2016), em que foi encontrada a aplicação desse processo. O detalhamento do Procnow-C, apresentado por Tasca (2013), motivou a escolha deste processo, no intuito de avaliar sua aplicação na condução da revisão deste trabalho.

O Proknow-C constitui-se de quatro etapas: a) seleção de um portfólio de artigos sobre o tema da pesquisa; b) análise bibliométrica do portfólio; c) análise sistêmica; d) definição da pergunta de pesquisa e objetivo de pesquisa. Neste trabalho, foram desenvolvidas as duas primeiras etapas do processo, com o objetivo de construir uma base para análise dos estudos e perspectivas de trabalhos futuros referentes à temática de visualização de ontologias.

Na Seção a seguir, são descritas as etapas do Proknow-C e os resultados de sua aplicação para o contexto deste artigo. 


\section{SELEÇÃO DO PORTFÓLIO BIBLIOGRÁFICO}

No contexto do Procknow-C, o portfólio bibliográfico constitui um conjunto restrito de publicações com reconhecimento científico, selecionados conforme o interesse de pesquisa e delimitações estabelecidas pelo pesquisador. Para isso, três atividades são necessárias: a) seleção do banco de artigos brutos; b) filtragem de artigos; c) teste de representatividade.

Para nortear a seleção do banco de artigos brutos, definiu-se a seguinte questão de pesquisa: "Qual é o estado da arte da visualização e navegação para ontologias, no contexto de pesquisa da Ciência da Informação?". Para essa questão, a expressão de busca escolhida para condução das pesquisas foi (Quadro 1):
Quadro 1 - Expressão de busca

$$
\text { (ontolog* } \text { visuali* }^{*} \text { OR (ontolog* }{ }^{*} \text { avigat*) }
$$

Fonte: Elaborado pelas autoras.

Para a escolha das bases de dados, foi utilizado o Portal de Periódicos Capes (http://www. periodicos.capes.gov.br). Na busca por área de conhecimento, foram escolhidas "Ciência Sociais Aplicadas" e a subárea "Ciência da Informação". Desta seleção, foram obtidas 29 bases de dados. As informações sobre as bases foram analisadas, e foram selecionadas aquelas que possuíam maior especificidade com a Ciência da Informação e abrangência de conteúdo. Dentro desse critério, foram selecionadas as bases: Information Science \& Technology Abstracts (ISTA); Library, Information Science \& Technology Abstracts (LISTA); Library and Information Science Abstracts (LISA); Web of Science e Scopus, detalhadas no Quadro 2.

Quadro 2 - Bases de dados consultadas e suas informações de conteúdo

\begin{tabular}{||l|l|}
\hline \multicolumn{1}{|c|}{ BASE DE DADOS } & \multicolumn{1}{|c|}{ INFORMAÇÕES SOBRE CONTEÚDOS } \\
\hline $\begin{array}{l}\text { Information Science E } \\
\text { Technology Abstracts (ISTA) }\end{array}$ & $\begin{array}{l}\text { Indexa artigos de mais de 450 publicações na área de ciência da informação, além } \\
\text { de livros, relatórios de pesquisa e anais de conferências e patentes, com cobertura } \\
\text { abrangente e contínua dos periódicos mais importantes nessa área. As datas de } \\
\text { cobertura remontam a meados da década de 1960. }\end{array}$ \\
\hline $\begin{array}{l}\text { Library, Information Science } \\
\text { E Technology Abstracts } \\
\text { (LISTA) }\end{array}$ & $\begin{array}{l}\text { Indexa mais de 500 periódicos científicos, além de livros e relatórios de pesquisas. } \\
\text { Esta base de dados também inclui o texto completo de mais de 240 periódicos } \\
\text { científicos. A cobertura de assuntos inclui biblioteconomia, classificação, } \\
\text { catalogação, bibliometria, recuperação de informações on-line, gestão de } \\
\text { informações e outros. A cobertura da base de dados remonta a meados dos anos } \\
1960 .\end{array}$ \\
\hline $\begin{array}{l}\text { Library and Information } \\
\text { Science Abstracts (LISA) }\end{array}$ & $\begin{array}{l}\text { Indexa mais de 400 títulos de periódicos de mais de 68 países e em mais de 20 } \\
\text { idiomas diferentes. As principais áreas de cobertura incluem: Inteligência artificial; } \\
\text { Tecnologia da informação, armazenamento e gestão; Tecnologia de Internet; } \\
\text { Gestão do conhecimento; Bibliotecas e arquivos; Gestão da informação; Estudos de } \\
\text { uso e de usuários; Informação biomédica; Sistemas de recuperação da informação; } \\
\text { Editoração; Telecomunicação e Internet. }\end{array}$ \\
\hline Web of Science & $\begin{array}{l}\text { Indexa somente os periódicos mais citados em suas respectivas áreas. É também } \\
\text { um índice de citações, informando, para cada artigo, os documentos por ele citados } \\
\text { e os documentos que o citaram. Possui, hoje, mais de 9000 periódicos indexados. }\end{array}$ \\
\hline Scopus & $\begin{array}{l}\text { Indexa resumos e citações da literatura científica e de fontes de informação de } \\
\text { nível acadêmico na Internet. Indexa mais de 21 mil periódicos, de cinco mil editores } \\
\text { internacionais, 24 milhões de patentes, além de outros documentos. }\end{array}$ \\
\hline
\end{tabular}

Fonte: Elaborado pelas autoras com informações extraídas do Portal Capes em janeiro de 2017. 
As pesquisas nas bases de dados foram realizadas em janeiro de 2017, utilizando-se os seguintes critérios de delimitação: i) Trabalhos publicados em periódicos científicos, livros e anais de conferências científicas; ii) Trabalhos publicados no período de 2007 a 2017.

O número total de trabalhos localizados nas pesquisas foi de 5514, distribuídos conforme o Gráfico 1. Analisando-se os resultados, observa-se que o elevado número de artigos retornados da base de dados Scopus (3444) e Web of Science (1997) ocorre pelo fato de essas bases serem multidisciplinares. O número reduzido de trabalho nas bases específicas LISA (49), LISTA (16) e ISTA (8) indicam que a temática tem sido pouco abordada no âmbito da Ciência da Informação.

Para a filtragem dessas referências, utilizou-se como suporte o software Endnote X7 (http://endnote.com/). A partir da importação das 5514 referências, foram excluídas 1013, identificadas pelo sistema como duplicadas. Além dessas, arbitrou-se por excluir as 330 referências do tipo Conference Review, recuperadas pela base de dados Scopus. Ao final, permaneceram para análise e observação um total de 4040 referências. A seguir, os títulos foram manualmente analisados e, no caso de estarem desalinhados com a questão de pesquisa, eles foram retirados do banco de referências. Com essa análise, filtraram-se 129 referências alinhadas com a questão de pesquisa deste artigo.

Gráfico 1 - Total de referências recuperadas nas bases de dados

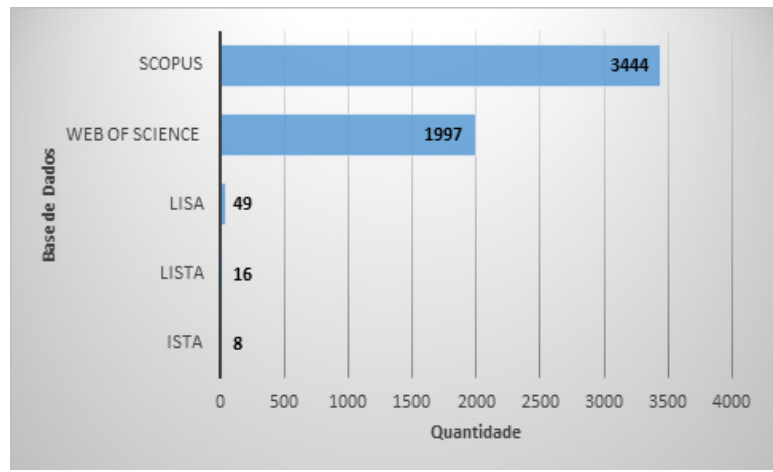

Fonte: Elaborado pelas autoras.
Conforme etapa prevista no Procknow-C, identificou-se o número de citações advindas do Google Acadêmico (https://scholar.google.com.br/) para cada uma das referências, estabelecendose um ranking de citações, com suporte do software Microsoft Excel. De forma arbitrária, considerou-se que as referências que estivessem dentro do percentual de $80 \%$ de participação do total de citações da amostra, seriam selecionadas para o portfólio bibliográfico. Com isso, 29 referências foram selecionadas por terem mais de 15 citações.

A partir de uma análise de ano e autores das 100 referências excluídas por esse processo, recuperaram-se mais 24 referências por terem sido publicadas por algum dos autores dos trabalhos mais citados ou porque foram publicadas em data recente.

Terminada essa etapa, buscou-se o artigo completo das 53 referências do Portfólio Bibliográfico. Por indisponibilidade do artigo no Portal Capes, excluíram-se quatro referências, e outras quatorze foram excluídas por não terem pertinência com a questão de pesquisa deste trabalho.

Para o alcance do objetivo desse trabalho considerou-se suficiente esse conjunto de 35 artigos selecionados e, portanto, não se realizou o teste de representatividade. A seguir apresentase a análise bibliométrica e a identificação de estudos e perspectivas de investigação referentes à visualização de ontologias.

\section{ANÁLISE BIBLIOMÉTRICA}

Conforme apresentado por Tasca (2013), a bibliometria prevista no Proknow-C tem por objetivo expandir o conhecimento do pesquisador, evidenciando os periódicos, autores, palavras-chaves e reconhecimento científico dos artigos do portfólio bibliográfico.

Analisando as fontes das referências selecionadas, identificou-se que $23 \%$ são títulos advindos de periódicos, $17 \%$ de capítulos de livros e outros $60 \%$ de conferências, conforme o Gráfico 2. 
Gráfico 2 - Fonte dos trabalhos do portfólio bibliográfico

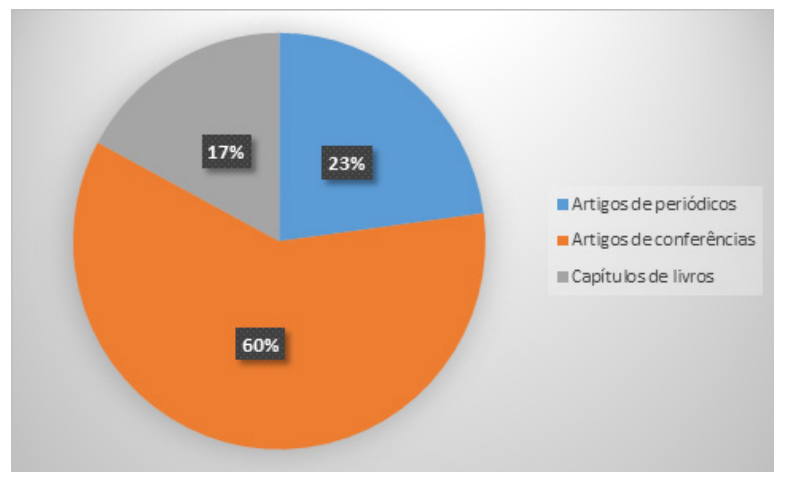

Fonte: Elaborado pelas autoras.

Com o objetivo de identificar os periódicos das referências do portfólio bibliográfico, elaborou-se uma lista de todos os títulos. O Gráfico 3 apresenta os títulos dos oito periódicos identificados, com o número de artigos do portfólio bibliográfico e o índice SCImago Journal \& Country Rank (SJR) (http:/ / www.scimagojr.com/). Nessa avaliação, destacou-se o Web Semantic (http://www. semantic-web-journal.net/) que se trata de um periódico multidisciplinar, com duas publicações sobre visualização de ontologias. Em relação à relevância científica, pelo índice SJR, o periódico ACM Computing Survey (http://csur.acm.org/) apresenta o maior indicador.

Gráfico 3 - Lista de Periódicos

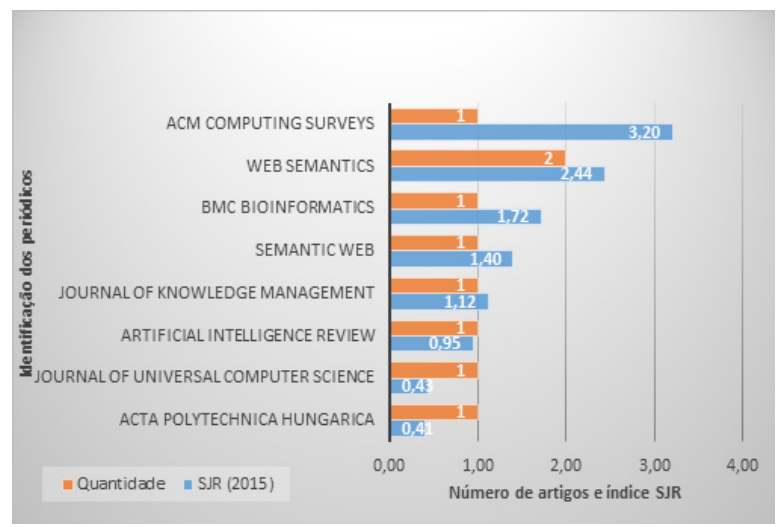

Fonte: Elaborado pelas autoras.
Para avaliação da relevância científica dos artigos, foi utilizada a medida de citações no Google Acadêmico. Nessa análise, destacase o artigo de Katifori et al (2007), com 408 citações. Outra menção que desperta atenção é o trabalho de Fu; Noy e Storey (2017), pela publicação ser a mais recente e já possuir 13 citações. O Gráfico 4, a seguir, apresenta as referências com mais de 13 citações, junto aos respectivos valores.

Gráfico 4 - Número de citações do Google Acadêmico

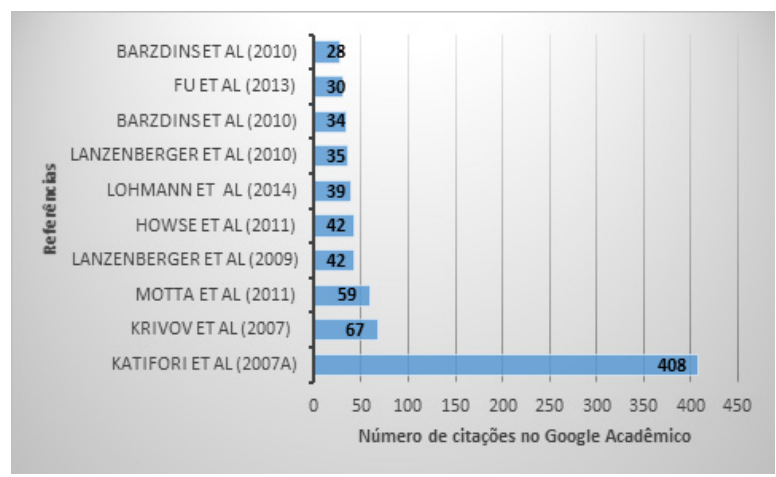

Fonte: Elaborado pelas autoras.

Em uma análise dos autores do portfólio, encontrou-se um conjunto de 123 autores. Entre eles, destacam-se treze autores com três ou mais artigos no conjunto do portfólio analisado, conforme apresenta o Gráfico 5.

Gráfico 5 - Lista de Autores e quantidade de artigos

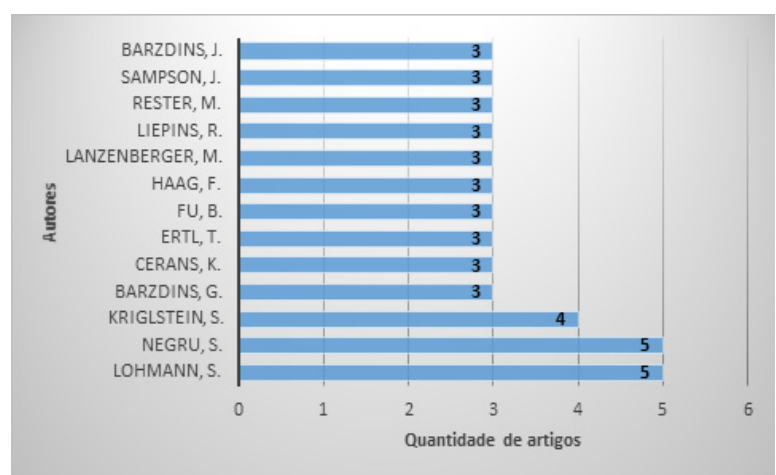

Fonte: Elaborado pelas autoras. 
Em uma pesquisa conduzida pelos autores Negru, S. e Lohmann, S., encontrou-se que se trata de pesquisadores, respectivamente, das instituições Alexandru Ioan Cuza University, na Romênia e da University of Stuttgart, na Alemanha.

Em uma última análise, foram avaliadas as palavras-chave utilizadas nos artigos selecionados do portfólio bibliográfico. Destacase do conjunto das 157 palavras identificadas, as cinco mais indicadas, conforme o Gráfico 6.

Gráfico 6 - Palavras chave e número de ocorrências

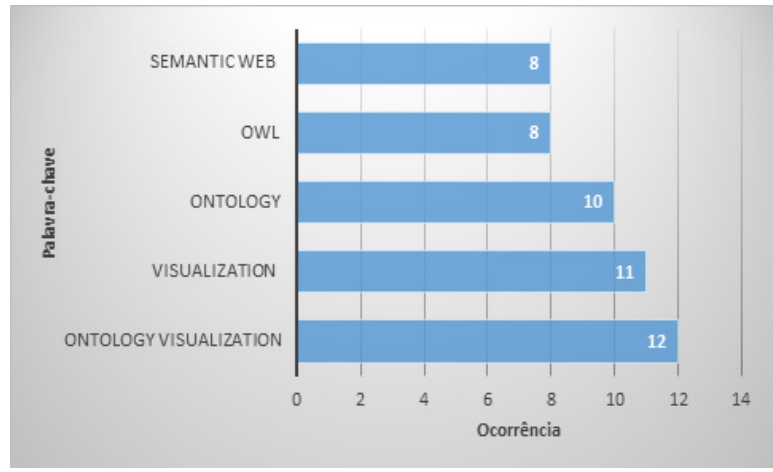

Fonte: Elaborado pelas autoras.

\section{ESTUDOS DE VISUALIZAÇÃO DE ONTOLOGIAS}

A partir da análise das 35 referências selecionadas, observou-se que os estudos realizados com o tema de visualização de ontologias podem ser categorizados em quatro tipos: 1) apresentação de ferramentas/protótipos; 2) comparação de ferramentas; 3) estudos com usuários; 4) revisão de literatura, distribuídos conforme apresenta o Gráfico 7.

Gráfico 7 - Áreas de estudos

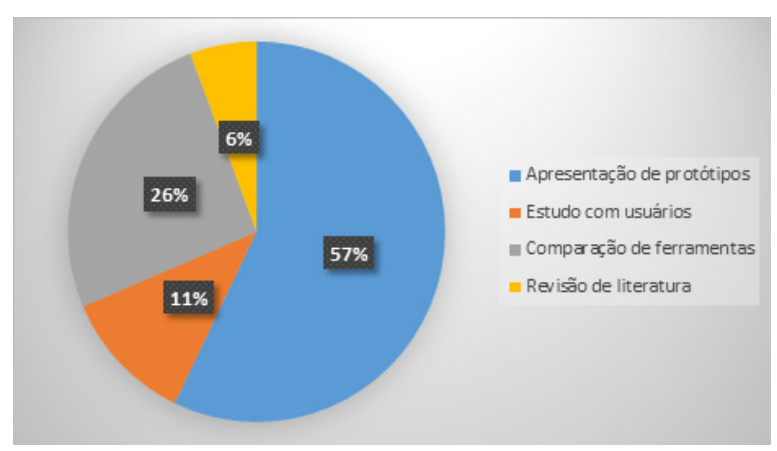

Fonte: Elaborado pelas autoras.

Nos trabalhos de apresentação de ferramentas/protótipos, presente em $57 \%$ do conjunto de referências analisadas observa-se que a comunidade científica está em busca de soluções para o problema de visualização de ontologias. Esses trabalhos se caracterizam pela descrição das funcionalidades das ferramentas/ protótipos, com relatos breves e estudos com usuários para avaliação comparativa com outras ferramentas ou protótipos. O Quadro 3, a seguir, apresenta um resumo das ferramentas relatadas na literatura, com as suas principais características e referências. 
Quadro 3 - Ferramentas e protótipos de visualização de ontologias

\begin{tabular}{|c|c|c|}
\hline $\begin{array}{c}\text { Ferramenta e } \\
\text { Disponibilidade }\end{array}$ & Características da visualização & Referências \\
\hline VOWL & $\begin{array}{l}\text { Baseada em grafos. A visualização é feita por uma } \\
\text { representação visual projetada para mapear os } \\
\text { elementos da OWL. Criada para usuários especialistas } \\
\text { e não-especialistas em ontologias. }\end{array}$ & $\begin{array}{l}\text { (LOHMANN et al, 2016) } \\
\text { (LOHMANN et al, 2014) } \\
\text { (LOHMANN; NEGRU; BOLD, 2014) } \\
\text { (NEGRU; LOHMANN, 2013) }\end{array}$ \\
\hline Knookcs & $\begin{array}{l}\text { A visualização é dedicada a representar as instâncias } \\
\text { relacionadas à estrutura nas ontologias. }\end{array}$ & $\begin{array}{l}\text { (KRIGLSTEIN; WALLNER, 2010) } \\
\text { (KRIGLSTEIN; WALLNER, 2011) } \\
\text { (KRIGLSTEIN, 2009) }\end{array}$ \\
\hline OntoSumViz & $\begin{array}{l}\text { Desenvolvida com base no uso de vetores descritivos } \\
\text { para gerar uma visualização que represente uma visão } \\
\text { geral de ontologia. Proposta para auxiliar a avaliação e } \\
\text { seleção de ontologias para um domínio. }\end{array}$ & (MACHOVÁ et al, 2016) \\
\hline MemoGraph & $\begin{array}{l}\text { Visualização desenvolvida visando ao uso universal } \\
\text { e voltada especificamente para pacientes com } \\
\text { Alzheimer. Adota uma representação com imagens } \\
\text { que podem ser escolhidas por usuários ou pelo } \\
\text { sistema. }\end{array}$ & (GHORBEL et al, 2016) \\
\hline OWLGrid & $\begin{array}{l}\text { Visualização gerada com uma notação baseada em } \\
\text { diagrama de classes em UML. }\end{array}$ & (CERANS et al, 2015) \\
\hline Osmosys & $\begin{array}{l}\text { Visualização com diferentes formatos para } \\
\text { representação dos conteúdos de } \\
\text { acesso via web. }\end{array}$ & (PSYLLIDIS, 2015) \\
\hline UML-like & $\begin{array}{l}\text { Visualização para uso no Protégé, baseado na } \\
\text { representação da linguagem UML. }\end{array}$ & (CAI; SHI; YANG, 2015) \\
\hline OLSViz & $\begin{array}{l}\text { Desenvolvida especificamente para visualização das } \\
\text { ontologias do repositório Ontology Lookup Service } \\
\text { (OLS). }\end{array}$ & $\begin{array}{l}\text { (VERCRUYSSE; VENKATESAN; } \\
\text { KUIPER, 2012) }\end{array}$ \\
\hline KC-Viz & $\begin{array}{l}\text { A visualização é gerada a partir de um método para } \\
\text { extração de conceitos chaves, usados para representar } \\
\text { as ontologias de forma concisa. }\end{array}$ & (MOTTA el al, 2011) \\
\hline OntoTrix & $\begin{array}{l}\text { Ferramenta projetada para permitir aos usuários } \\
\text { visualizar e navegar em conjuntos de instâncias e suas } \\
\text { relações em ontologias. }\end{array}$ & (BACH et al, 2011) \\
\hline SSN-XG & $\begin{array}{l}\text { Visualização baseada em representação de diagramas } \\
\text { conceituais. }\end{array}$ & (HOWSE et al, 2011) \\
\hline OWLGrEd & $\begin{array}{l}\text { Ferramenta para edição e visualização de ontologias, } \\
\text { com uma representação gráfica baseada na linguagem } \\
\text { UML. }\end{array}$ & $\begin{array}{l}\text { (BARZDINS et al, 2010a) } \\
\text { (BARZDINS et al, 2010b) }\end{array}$ \\
\hline OWLEasyViz & $\begin{array}{l}\text { Editor de ontologia que combina uma representação } \\
\text { textual e gráfica de ontologias, com uma proposta de } \\
\text { interface para atender usuários especialistas e não- } \\
\text { especialistas em ontologias. }\end{array}$ & $\begin{array}{l}\text { (CATENAZZI; SOMMARUGA; } \\
\text { MAZZA, 2009) }\end{array}$ \\
\hline GrOWL & Visualização baseada em grafos para uso no Protégé. & (KRIVOV; WILLIAMS; VILLA, 2007) \\
\hline
\end{tabular}

Fonte: Elaborado pelas autoras. 
Quanto aos estudos relacionados à avaliação de ferramentas, encontrou-se o relato em $9 \%$ das referências do portfólio bibliográfico, sendo o estudo mais amplo o de Katifori et al (2007), sendo esta, ainda, a referência mais citada do conjunto. Outros trabalhos que também apresentaram estudos comparativos de ferramentas são: Lambrix et al (2016), Schaaf et al (2016), Ramakrishnan e Vijayan (2014), Lanzenberger; Sampson e Rester $(2009,2010)$, Lanzenberger et al (2008) e Katifori et al (2008). Entre esses estudos, destaca-se o de Haag et al (2014), que propõe um benchmarck para avaliação das ferramentas de visualização de ontologias.

Nos trabalhos relacionados a estudos com usuários encontraram-se relatos de estudos de avaliação de usabilidade, comparando técnicas de visualização baseadas em lista identada e grafos. Nessa categoria encontram-se os trabalhos de Fu; Noy e Storey (2017), Huynh e Fu (2016), Fu; Noy e Storey (2013). Em uma perspectiva de estudo de usuários voltado para especificação de requisitos para visualização de ontologias, há Kriglstein (2009).

Em estudo de revisão de literatura foram encontrados Saghafi (2016) e Mikhailov; Petrov e Lantow (2016). Saghafi (2016), é um estudo que busca analisar os métodos de visualização de ontologias relatados na literatura e correlacionar os resultados dos estudos comparativos. No trabalho de Mikhailov; Petrov e Lantow (2016), relatam-se os resultados de uma revisão sistematizada, usando o método Structured Literature Analysis (SLA) de Kitchenham (2004), que teve como objetivo analisar os trabalhos publicados sobre o assunto em quatro conferências internacionais.

\section{PERSPECTIVAS DE TRABALHOS FUTUROS}

A partir da análise dos trabalhos selecionados no portfólio bibliográfico deste estudo, foram identificados quatro campos de investigação abertos a desenvolvimento de trabalhos futuros em visualização de ontologias: i) visualização de ontologias complexas, com centenas de elementos; ii) ferramentas para usuários leigos em ontologias; iii) estudos de usabilidade e de requisitos de usuários; iv) contribuição cognitiva para usuários.

A visualização de ontologias complexas, conforme apresenta Lohmann et al (2016), ainda é uma questão que dificulta o desenvolvimento de interface das ferramentas. As representações grá- ficas, em geral, são viáveis até certo tamanho de ontologias. Quando não se torna possível obter uma visão geral, perde-se de certa forma benefício de compreender a organização dos conteúdos. No estudo de avaliação de ferramentas, Schaaf et al (2016) concluíram que nenhuma das ferramentas analisadas por ele era adequada para visualização de grandes ontologias. No trabalho de Machová et al (2016), relata-se uma investigação nessa direção, buscando uma representação para melhorar a navegação do usuário no espaço ontológico com ontologias de numerosos elementos.

O desenvolvimento de ferramentas para usuários com pouca experiência em ontologias pode fornecer novas perspectivas para o uso dessas, proporcionando compartilhamento de conhecimento de domínios e novos insumos para o desenvolvimento e aprimoramento das representações. Conforme Ghorbel et al (2016), existem muitas ferramentas de visualização de ontologias disponíveis na literatura. No entanto, apenas alguns deles são dedicados a usuários não familiarizados com ontologias. Além disso, os autores destacam que não identificaram uma ferramenta de visualização de ontologia que siga a filosofia do "design para todos". Essa filosofia consiste em considerar não só os usuários inteligentes saudáveis que dominam as tecnologias, mas todos, de todas as idades e com diferentes níveis de habilidades, incluindo, obviamente, os idosos que sofrem de doença de doenças degenerativas, como o Alzheimer.

Os estudos de usabilidade e de requisitos de usuários para visualização de ontologias, conforme apresenta Fu et al (2017), necessitam ser realizados e aprofundados para maior compreensão dos benefícios, da eficiência e da eficácia das técnicas de visualização, bem como para o desenvolvimento de novas funcionalidades para as ferramentas. Em kriglstein (2009) e Fu; Noy e Storey (2013), sugere-se que o desenvolvimento das ferramentas seja feito em contínuo acompanhamento e avaliação de usuários.

No aspecto de contribuição cognitiva para os usuários de visualização de ontologias, percebe-se que, embora os estudos de desenvolvimento de ferramentas visem favorecer a cognição humana, há uma carência de estudos mais aprofundados para avaliação e medida dessa contribuição.

A apresentação dessas perspectivas não esgota a ainda emergente necessidade dos sistemas de recuperação de informação, da web semântica e, ainda, dos diversos domínios de conhecimento, tais como da saúde, da educação e do governo. 


\section{CONSIDERAÇÕES FINAIS}

Este artigo apresentou uma revisão sistematizada da literatura referente ao tema Visualização de Ontologias. Utilizando-se de etapas do processo Procknow-C, selecionouse um conjunto de 35 referências científicas, publicadas entre 2007 e 2017, em uma coleção chamada portfólio bibliográfico. A relevância dos resultados desse trabalho consiste na organização do estudo de estado da arte nas pesquisas de visualização de informação aplicada às ontologias. A partir dos resultados, tem-se uma visão das principais temáticas de estudos já realizadas e as tendências encontradas de rumos de novas investigações, proporcionando aos pesquisadores avançarem em definições de problemas e metodologias de pesquisa. Além disso, a identificação dos principais periódicos, conferências, autores e bases de dados, poderá favorecer a busca por novos trabalhos.

Pela análise bibliométrica do estudo, identificou-se que $60 \%$ das referências selecionadas para o estudo são de artigos apresentados em conferências, indicando um crescente interesse da comunidade científica em discutir o tema. Quanto às fontes publicadas em periódicos, destacaram-se o Web Semantic e o ACM Computing Survey. O primeiro trata-se de um periódico multidisciplinar e com duas publicações sobre visualização de ontologias, e o segundo é considerado de maior relevância científica pelo índice SJR.

Em uma análise aprofundada dos conteúdos foram identificados quatro tipos principais de abordagens do tema: apresentação de ferramentas/protótipos, comparação de ferramentas, estudos com usuários e revisão de literatura, sendo 57\% dos trabalhos destinados à apresentação de ferramentas/protótipos. Quanto a perspectivas de trabalhos futuros em visualização de ontologias, destacaram-se quatro temáticas: visualização de ontologias complexas, com centenas de elementos; ferramentas para usuários leigos em ontologias; estudos de usabilidade e de requisitos de usuários; contribuição cognitiva para usuários.

Quanto à adoção do processo Proknow-C, considerou-se que suas etapas bem definidas para seleção do portfólio bibliográfico, favorecem a organização da revisão sistemática. No entanto, trata-se de uma seleção trabalhosa, que requer o auxílio de softwares gerenciadores de referências e disposição para manipulação e verificação de informações.

Por fim, destaca-se a relevância do tema de visualização de ontologias apresentada em todos os estudos, visto o potencial para melhorar tarefas como a compreensão humana do conhecimento implícito, bem como o alinhamento da informação em domínios de aplicação.

Artigo recebido em 2I/03/2017 e aceito para publicação em II/09/20I7

\section{ONTOLOGY VISUALIZATION: studies and perspectives}

ABSTRACT This study presents the results of a systematic review of literature that aimed to analyze the studies and prospects of further work on visualization of ontologies. The methodology was developed upon the application of the Proknow-C process steps (Knowledge Development Process - Constructivist) in order to select a portfolio of 35 bibliographic scientific references, published from 2007 to 2017. Among the results of the analysis four major approaches have been identified as a study's main theme: presentation of tools and prototypes; comparison of tools; studies with users and literature review, being the case that $57 \%$ of the selected works were intended for the presentation of tools/prototypes. In regard to the prospects for further work as visualization of ontologies, four topics are highlighted: complex ontologies, where there are hundreds of elements; tools for a layman in ontologies; usability studies, requirements of users and evaluation of cognitive contribution to users. The contribution of this work is based on the outline left to future research and legitimation of the systematic review process as a generator of knowledge.

Keywords: Information Visualization. Ontologies. Systematic review. 


\section{REFERÊNCIAS}

ALMEIDA, M. B.; BAX, M. P. Uma visão geral sobre ontologias: pesquisa sobre definições, tipos, aplicações, métodos de avaliação e de construção. Ciência da Informação, Brasilia, DF, v. 32, n. 3, p. 7-20, set./dez. 2003. Disponível em: <http://www.scielo.br/pdf/ci/ v32n3/19019.pdf>. Acesso em: 20 mar. 2017.

$\mathrm{BACH}, \mathrm{B}$. et al. OntoTrix: a hybrid visualization for populated ontologies. In: INTERNATIONAL CONFERENCE COMPANION ON WORLD WIDE WEB, 20., 2011, Hyderabad, India. Proceedings... New York: ACM, 2011. p. 177-180.

BĀRZDIN̦Š, J. et al. OWLGrEd: a UML style graphical notation and editor for OWL 2 . In: INTERNATIONAL WORKSHOP OWL: EXPERIENCE AND DIRECTIONS, 7., 2010, Proceedings... 2010a. Disponível em: <http:// webont.org/owled/2010/ papers / owled2010_ submission_5.pdf>. Acesso em: 20 mar. 2017.

UML style graphical notation and editor for OWL 2. In: INTERNATIONAL CONFERENCE ON PERSPECTIVES IN BUSINESS INFORMATICS RESEARCH, 9., 2010b, Proceedings... Rostock, Germany: Springer, 2010. p. 102-114.

CAI, Z.; SHI, K.; YANG, H. A novel visualization for ontologies of semantic web representation. In: INTERNATIONAL CONFERENCE ON COMPUTATIONAL INTELLIGENCE AND COMMUNICATION NETWORKS, 2015, Jabalpur, Proceedings... Jabalpur: IEEE, 2015. p. 1371-1374. Electronic ISSN: 2472-7555.

CAMPOS, M. L. A. O papel das definições na pesquisa em ontologia. Perspectivas em Ciência da Informação, Belo Horizonte, v. 15, n. 1, p. 220-238, jan./abr. 2010. Disponível em: <http:// www.scielo.br/pdf/pci/v15n1/13.pdf>. Acesso em: 20 mar. 2017.

CARD, Stuart K.; MACKINLAY, Jock D.; SHNEIDERMAN, Ben. Readings in information visualization: using vision to think. San Francisco: Morgan Kaufmann, 1999. 686 p.

CARD, Stuart K.; ROBERTSON, George G.; MACKINLAY, Jock D. The information visualizer, an information workspace. In: SIGCHI CONFERENCE ON HUMAN FACTORS IN COMPUTING SYSTEMS, 1991, New Orleans. Proceedings... New York: ACM Press, 1991. p. 181-186. ISBN: 0-89791-383-3.

CATENAZZI, N.; SOMMARUGA, L.; MAZZA, R. User-friendly ontology editing and visualization tools: the OWLeasyViz approach. In: INTERNATIONAL CONFERENCE INFORMATION VISUALISATION, 13., 2009, Barcelona. Proceedings... Los Alamitos: IEEE, 2009. p. 283-288.

CERANS, K. et al. Domain-specific OWL ontology visualization with OWLGrEd. In: EXTENDED SEMANTIC WEB CONFERENCE, 2012, Heraklion. Proceedings... Heidelberg: Springer, 2012. p. 419-424. ISBN: 978-3-662-466407 (Print). 978-3-662-46641-4 (Online).

CORREA, R. F.; VIEIRA, J. M. de L. Representações visuais para recuperação de informação na BDTD-UFPE. Perspectivas em Ciência da Informação, v.18, n.4, p.18-34, out./ dez. 2013. Disponível em: <http://www.scielo. br/pdf/pci/v18n4/03.pdf>. Acesso em: 20 mar. 2017.

ENSSLIN, L. et al. Processo para evidenciação do estado da arte aplicado ao tema gestão de produção científica. Transinformação, v. 27, n. 3, p. 219-228, 2015. Disponível em: <http:// www.scielo.br/pdf/tinf/v27n3/0103-3786tinf-27-03-00219.pdf>. Acesso em: 20 mar. 2017.

ENSSLIN, L.; ENSSLIN, S.; PACHECO, G. C. Um estudo sobre segurança em estádios de futebol baseado na análise da literatura internacional. Perspectivas em Ciências da Informação, v. 17, n. 2, p. 71-91, 2012.

FEINER, S. K.; BESHERS, C. Worlds within worlds: metaphors for exploring n-dimensional virtual worlds. In: ANNUAL ACM SIGGRAPH SYMPOSIUM ON USER INTERFACE SOFTWARE AND TECHNOLOGY, 3., 1990, Snowbird. Proceedings... Snowbird: ACM, 1990. p. 76-83. Disponível em: <http:// citeseerx.ist.psu.edu/viewdoc/download? doi=10.1.1.34.9201\&rep=rep1\&type $=$ pdf $>$. Acesso em: 20 mar. 2017. 
FREITAS, C. M. D. S. et al. Introdução à visualização de informações. RITA - Revista de Informática Teórica e Aplicada, Porto Alegre, v. 8, n. 2, p. 143-158, out. 2001.

FU, B.; NOY, N. F.; STOREY, M.-A. Eye tracking the user experience: an evaluation of ontology visualization techniques. Semantic Web, v. 8, n. 1, p. 23-41, 2017.

Indented tree or graph? a usability study of ontology visualization techniques in the context of class mapping evaluation. In: INTERNATIONAL SEMANTIC WEB CONFERENCE, 12., 2013, Sydney. Proceedings... Heidelberg: Springer, 2013. p. 117-134.

GHORBEL, F. et al. MEMO GRAPH: an ontology visualization tool for everyone. Procedia Computer Science, v. 96, p. 265-274, 2016. Disponível em: <https:/ /www.researchgate.net/ publication/307914732_MEMO_GRAPH_An_ Ontology_Visualization_Tool_for_Everyone/ fulltext/57d2795108ae6399a38d24af/307914732_ MEMO_GRAPH_An_Ontology_Visualization_ Tool_for_Everyone.pdf?origin=publication detail>. Acesso em: 20 mar. 2017.

GIL, A. C. Como elaborar projetos de pesquisa. 5. ed. São Paulo: Atlas, 2010. 184 p.

GRUBER, T. R. A translation approach to portable ontology specifications. Knowledge acquisition, Stanford, v. 5, n. 2, p. 199-220, 1993. Disponível em: <http://tomgruber.org/writing/ ontolingua-kaj-1993.pdf>. Acesso em: 20 mar. 2017.

GUIZZARDI, G. Desenvolvimento para e com reuso: um estudo de caso no domínio de vídeo sob demanda. 2000. 202 f. Dissertação (Mestrado em Informática)- Universidade Federal do Espírito Santo, Vitória, 2000. Disponível em: <http:/ / www.inf.ufes.br/ falbo/download/ aulas/es-m/2007-1/dissertacao_gian.pdf $>$. Acesso em: 20 mar. 2017.

HAAG, F. et al. OntoViBe: an ontology visualization benchmark. In: INTERNATIONAL CONFERENCE ON KNOWLEDGE ENGINEERING AND KNOWLEDGE MANAGEMENT: VISUAL, 19., 2014, Linköping.
Proceedings... Gewerbestrasse: Springer International Publishing, 2014. p. 14-27. eBook ISBN: 978-3-319-17966-7.

HOWSE, J. et al. Visualizing ontologies: a case study. In: INTERNATIONAL SEMANTIC WEB CONFERENCE, 10., 2011, Bonn. Proceedings... Heidelberg: Springer-Verlag, 2011. p. 257-272.

HUYNH , A; FU, B. Information search in ontology visualization - an eyetracking user study of indented list on desktop and tablet computers. In: INTERNATIONAL JOINT CONFERENCE ON KNOWLEDGE DISCOVERY, KNOWLEDGE ENGINEERING AND KNOWLEDGE MANAGEMENT, 8., 2016, Porto. Proceedings... Setúbal: Science and Technology Publications, 2016. p. 129-135.

KATIFORI, A. et al. Ontology visualization methods: a survey. ACM Computing Surveys, v. 39, n. 4, p. 10, 2007. Disponível em : <http:// disi.unitn.it/ p2p/RelatedWork/Matching/a10katifori.pdf>. Acesso em : 20 mar. 2017.

study of four ontology visualization methods for information retrieval tasks. In: INTERNATIONAL CONFERENCE ON RESEARCH CHALLENGES IN INFORMATION SCIENCE, 2., 2008, Marraquexe. Proceedings... Nice: IEEE, 2008. p. 133-140.

KITCHENHAM, B. Procedures for performing systematic reviews. Keele, UK, Keele University, v. 33, 2004, p. 1-26, 2004.

KRIGLSTEIN, S. User requirements analysis on ontology visualization. In: INTERNATIONAL CONFERENCE ON COMPLEX, INTELLIGENT AND SOFTWARE INTENSIVE SYSTEMS, 2009, Fukuoka. Proceedings... Los Alamitos: IEEE, 2009. p. 694-699.

KRIGLSTEIN, S.; WALLNER, G. Development process and evaluation of the ontology visualization tool knoocks-a case study. In: INTERNATIONAL CONFERENCE ON IMAGING THEORY AND APPLICATIONS AND INTERNATIONAL CONFERENCE ON INFORMATION VISUALIZATION THEORY AND APPLICATIONS, 2011, Vilamoura. 
Proceedings... Setúbal: Science and Technology Publications , 2011. p. 187-197.

KRIGLSTEIN, S.; WALLNER, G. Knoocks-a visualization approach for owl lite ontologies. In: INTERNATIONAL CONFERENCE ON COMPLEX, INTELLIGENT AND SOFTWARE INTENSIVE SYSTEMS, 4., 2010, Cracóvia, Proceedings... IEEE, 2010. p. 950-955.

KRIVOV, S.; WILLIAMS, R.; VILLA, F. GrOWL: a tool for visualization and editing of OWL ontologies. Web Semantics: Science, Services and Agents on the World Wide Web, v. 5, n. 2, p. 54-57, 2007.

LAMBRIX, P. et al. Visualization for ontology evolution. In: INTERNATIONAL WORKSHOP ON VISUALIZATION AND INTERACTION FOR ONTOLOGIES AND

LANZENBERGER, M. et al. Visual ontology alignment for knowledge sharing and reuse. Journal of Knowledge Management, v. 12, n. 6, p. 102-120, 2008.

LANZENBERGER, M.; SAMPSON, J.; RESTER, M. Ontology visualization: tools and techniques for visual representation of semi-structured meta-data. J. UCS, v. 16, n. 7, p. 1036-1054, 2010.

Visualization in ontology tools. In: INTERNATIONAL CONFERENCE ON COMPLEX, INTELLIGENT AND SOFTWARE INTENSIVE SYSTEMS, 2009. Fukuoka. Proceedings... Los Alamitos: IEEE, 2009. p. 705-711.

LOHMANN, S. et al. Visualizing ontologies with VOWL. Semantic Web, v. 7, n. 4, p. 399-419, 2016. Disponível em: <http://www.semanticweb-journal.net/system/files/swj1114.pdf >. Acesso em: 20 mar. 2017.

VOWL 2: user-oriented visualization of ontologies. In: INTERNATIONAL CONFERENCE ON KNOWLEDGE ENGINEERING AND KNOWLEDGE MANAGEMENT, 19., 2014, Linköping. Proceedings... Gewerbestrasse: Springer International Publishing, 2014. p. 266-281. eBook ISBN: 978-3-319-13704-9. DOI: 10.1007/978-3-31913704-9_21.
LOHMANN, S.; NEGRU, S; BOLD, D. The ProtégéVOWL plugin: ontology visualization for everyone. In: EUROPEAN SEMANTIC WEB CONFERENCE, 11., 2014, Anissaras. Proceedings... Basel: Springer International Publishing, 2014. p. 395-400. DOI: 10.1007/978-3319-11955-7_55.

MACHADO, K. C. et al. Avaliação de desempenho na gestão da informação digital: contribuições da literatura científica. Inf. \& Soc.:Est., João Pessoa, v.26, n.2, p. 155-172, maio/ ago. 2016

MACHOVÁ, K. et al. Ontology evaluation based on the visualization methods, context and summaries. Acta Polytechnica Hungarica, v. 13, n. 4, p. 53-76, 2016. Disponível em: <https://www.uni-obuda.hu/journal/ Machova_Vrana_Mach_Sincak_68.pdf $>$. Acesso em: 20 mar. 2017.

MARCONI, M. A.; LAKATOS, E. M. Fundamentos da metodologia científica. 7. Ed. São Paulo: Altas, 2010.

Fundamentos de metodologia científica.

5. ed. São Paulo: Atlas, 2003.

MOTTA, E. et al. A novel approach to visualizing and navigating ontologies. In: INTERNATIONAL SEMANTIC WEB CONFERENCE, 10., 2011, Bonn. Proceedings... Heidelberg: SpringerVerlag, 2011. p. 470-486. eBook ISBN: 978-3-64225093-4. DOI: 10.1007/978-3-642-25073-6_30.

NEGRU, S.; LOHMANN, S. A Visual notation for the integrated representation of OWL ontologies. In: INTERNATIONAL CONFERENCE ON WEB INFORMATION SYSTEMS AND TECHNOLOGIES, 9., 2013, Aachen. Proceedings... Setúbal: Science and Technology Publications, 2013. p. 308-315. ISBN: 978-9898565-54-9. DOI:10.5220/0004373003080315.

PSYLLIDIS, A. OSMoSys: a web interface for graph-based RDF data visualization and ontology browsing. In: INTERNATIONAL CONFERENCE ON WEB ENGINEERING, 15.,

RAMAKRISHNAN, S.; VIJAYAN, A. A study on development of cognitive support features 
in recent ontology visualization tools. Artificial Intelligence Review, v. 41, n. 4, p. 595-623, 2014.

SAGHAFI, A. Visualizing ontologies: a literature survey. In: INTERNATIONAL CONFERENCE ON CONCEPTUAL STRUCTURES, 22., Annecy. Proceedings... Springer International Publishing, 2016. p. 204-221. Print ISBN: 978-3-319-40984-9. Online ISBN: 978-3-319-40985-6.

SCHAAF, M. et al. Visualization of large ontologies in university education from a tool point of view. Studies in health technology and informatics, v. 228, p. 349-353, 2016. DOI: 10.3233/978-1-61499-678-1-349.

SHNEIDERMAN, B. The eyes have it: A task by data type taxonomy for information visualizations. In: SYMPOSIUM ON VISUAL LANGUAGES. 1996, Boulder. Proceedings... Los Alamitos: IEEE Computer Society Press, 1996. p. 336-343. Disponível em: <http://www. interactiondesign.us / courses / 2011_AD690/ PDFs/Shneiderman_1996.pdf>. Acesso em: 20 mar. 2017. Print ISSN: 1049-2615. DOI: 10.1109/ VL.1996.545307.

TASCA, J. E. A contribuição da avaliação de desempenho, como um instrumento de apoio à decisão, para a prevenção ao crime baseada no ambiente. 350 f. Tese (Doutorado em Engenharia de Produção)- Universidade Federal de Santa Catarina, Florianópolis, 2013.

TASCA, J. et al. An approach for selecting a theoretical framework for the evaluation of training programs. Journal of European Industrial Training, v. 34, n. 7, p. 631-655, 2010.

VICKERY, B. C. Ontologies. Journal of information science, v. 23, n. 4, p. 277-286, 1997.

VERCRUYSSE, S.; VENKATESAN, A.; KUIPER, M. OLSVis: an animated, interactive visual browser for bio-ontologies. BMC bioinformatics, v. 13, n. 116. p. 1-9, 2012. Disponível em: <https://www.ncbi.nlm.nih.gov/pmc/articles/ PMC3394205/pdf/1471-2105-13-116.pdf >. Acesso em: 20 mar. 2017. DOI: 10.1186/14712105-13-116. 